\title{
Intercultural Competence of Western Teachers for Nepalese Rescuers
}

\author{
Monika Brodmann Maeder, ${ }^{1,2}$ Raphael Saghir, ${ }^{3}$ Matiram Pun, ${ }^{1}$ Agnieszka Elzbieta Stawinoga, ${ }^{4}$ \\ Rachel Turner, ${ }^{1}$ Giacomo Strapazzon, ${ }^{1}$ Aristomenis K. Exadaktylos, ${ }^{2}$ and Hermann Brugger ${ }^{1}$
}

\begin{abstract}
Brodmann Maeder, Monika, Raphael Saghir, Matiram Pun, Agnieszka Elzbieta Stawinoga, Rachel Turner, Giacomo Strapazzon, Aristomenis K. Exadaktylos, and Hermann Brugger. Intercultural competence of Western teachers for Nepalese rescuers. High Alt Med Biol. 20:22-27, 2019.

Introduction: Educational projects in mountain rescue in Nepal have a long tradition. They are usually led by Western experts who train their Nepalese colleagues using teams of people with diverse cultural background. To better understand the challenges of these encounters, we conducted a prospective cohort study during the first mountain rescue instructor course in Nepal.

Methods: Western instructors (WIs) and Nepalese instructor candidates (NICs) were asked to self-assess their intercultural competence with the help of questionnaires. The responses were compared and analyzed for differences between WIs and NICs and differences in a pre-post assessment of the WIs. In addition, semistructured interviews were conducted with randomly selected NICs.

Results: We found significant differences in communication styles between NICs and WIs: NICs showed a preference to establish relationships before discussing business and not to speak openly in conflict situations. WIs were much more direct and preferred dispassionate exchanges. In an assessment after the course, WIs had changed their attitude toward the host culture.

Conclusions: We found differences in communication styles between WIs and NICs that are relevant to globalized medical education. Faculty members should be prepared before implementing medical training abroad and should have time to experience the host culture.
\end{abstract}

Keywords: education; faculty development; Himalayas; intercultural competence; mountain rescue

\section{Introduction}

\section{Mountain rescue training in Nepal}

$\mathbf{E}$ DUCATIONAL PROJECTS in the field of mountain rescue involving Nepalese students and foreign ("Western") instructors have been in place since 2009 (Maeder et al., 2014; Thapa et al., 2014). The field of education in mountain emergency medicine has consistently aimed to introduce and adapt urban medical standards in mountain rescue situations. A milestone for the field was the establishment of the International Commission for Mountain Emergency Medicine (ICAR MEDCOM), which made a transition in the early 2000s to transfer know-how from Western Mountain Rescue
Services to the rest of the world, focusing on theoretical and applied training based upon evidence-based recommendations (Ellerton et al., 2009; Strapazzon et al., 2011; Tomazin et al., 2011). The majority of these initiatives have focused on the training of Nepalese rescuers and physicians, facilitated by Western instructors (WIs). The first instructor course took place in 2015, after several years of training Nepalese rescuers. The goal of this pilot instructor course for Nepalese rescuers and rescue doctors in 2015 was to create a Nepalese faculty of capable instructors, who would themselves be able to conduct mountain rescue training initiatives independently with future candidates. The WIs chosen to attend were all experienced teachers and mountain rescue specialists from

\footnotetext{
${ }^{1}$ Institute of Mountain Emergency Medicine, EURAC Research, Bolzano, Italy.

${ }^{2}$ Department of Emergency Medicine, Inselspital, Bern University Hospital, University of Bern, Bern, Switzerland.

${ }^{3}$ University of Bern, Faculty of Medicine, Bern, Switzerland.

${ }^{4}$ Management and Committees, EURAC Research, Bolzano, Italy.
}

(c) Monika Brodmann Maeder et al., 2018; Published by Mary Ann Liebert, Inc. This Open Access article is distributed under the terms of the Creative Commons Attribution Noncommercial License (http://creativecommons.org/licenses/by-nc/4.0/) which permits any noncommercial use, distribution, and reproduction in any medium, provided the original author(s) and the source are cited. 
Europe and United States, with differing prior knowledge of or exposure to Nepalese culture. We refer to them as WIs. The Nepalese students or instructor candidates (NICs) had participated in at least one prior internationally led training course and were invited to become the first Nepalese mountain rescue instructors.

\section{Medical education in a globalized world}

The growing mobility of our society and exponential advances in communication and technology have influenced education and how education may be delivered. However, despite the advent of advanced global interaction, education in different cultural or regional areas is challenging (Emert and Pearson, 2007). Various terms coexist in the literature describing different examples of such teaching-learning activities. Some publications use the term intercultural, others use transcultural, cross-cultural education, or they talk about cultural competence or sensitivity (Volet, 2004; Sinicrope et al., 2007; Kratzke and Bertolo, 2013; Alizadeh and Chavan, 2016). In line with the term "cultural competence," within this study we chose to accept the definition of intercultural competence as the ability to work and communicate effectively and appropriately with people from culturally different backgrounds (Deardorff, 2009). Most of the published articles on intercultural education focus on the challenges of intercultural exchanges (Jha et al., 2015), and identify the level of difficulty associated with comprehensive assessment of behavioral indices that may determine intercultural competence or sensitivity (Deardorff, 2006; Drain et al., 2007; Hamilton, 2009; Holmes and O'Neill, 2012; Al Ansari et al., 2015).

\section{Research question}

Based on prior personal experience of the lead author, we wanted to assess intercultural competences of expert WIs and Nepalese rescuers and rescue doctors during the first mountain rescue instructor course in Nepal. Our primary research question focused upon whether different communication styles can be observed between instructors and course participants and whether this may influence teaching and learning experiences.

\section{Methods}

\section{Setting}

In 2015-4 months after the devastating Gorkha earthquake (M. B. Maeder \& Pun, 2018; Zafren et al., 2018) —an international group of experts in mountain medicine organized a 10-day instructor course in Kathmandu for Nepalese mountain rescuers and physicians with special competence in mountain rescue medicine. During this course, a prospective cohort study was conducted with the goal to identify the intercultural competence of both WIs and NICs.

\section{Data collection}

The primary data collection employed self-assessment of intercultural competence of all participants, utilizing an adapted form of the "Assessing Intercultural Competencies (AIC) questionnaire" (Fantini and Tirmizi, 2006). This questionnaire was originally developed by Fantini (2009) within a project of the Federation of The Experiment in
International Living (FEIL) (July 2005-December 2006). The original version of the AIC was designed for the assessment of intercultural outcomes on participants and their hosts in select civic service programs. It can be utilized free of charge and is openly accessible through the World Learning at DigitalCollections@SI. As we were not able to find a validated questionnaire that was designed for our setting, we decided to use the FEIL AIC questionnaire and adapted it to the needs of our project. Owing to the different setting for our course in Nepal, we had to change the respective country and language and deleted some of the questions that were not applicable, for example, questions about host families. The rest of the questions were maintained.

WIs and NICs were asked to complete distinct questionnaires in English. WIs were asked to complete a questionnaire before the course (WI pretest) (Supplementary Appendix SA1; Supplementary Data are available online at www.liebertpub.com/ham) and a questionnaire at the end of the course (WI post-test) (Supplementary Appendix SA2). The NICs completed a single questionnaire during the course (NIC test) (Supplementary Appendix SA3). In addition, six NICs were interviewed by phone after the course who answered questions from the Nepalese coinvestigator (M.P.) in their own native language. Primary data were obtained separately for the WIs and for the NICs, after having received a written and informed consent from each participant. During the 10-day instructor course in Kathmandu, Nepal, one investigator (R.S.) was dedicated full-time to data retrieval and entry. The study was approved by the ethical committee of Bolzano/Italy (Prot. 0116946-BZ).

\section{Demographic data}

Demographic factors such as age, gender, and nationality of the subjects were recorded. In addition, the following information was collected: religion, native language, other languages, and intercultural experience of the WIs with a special focus on prior visits to Nepal and other Asian countries.

\section{WI pretest}

The WI pretest consisted of four separate sections: the first section consisted of demographic set of questions about the participant, the second focused on personal characteristics, the third on communication styles, and the fourth was a self-evaluation of the participant's personal intercultural abilities. The total number of questions of all four sections was 145 .

\section{WI post-test}

The WI post-test consisted of five separate sections. The first section covered personal characteristics, the second motivation and options, the third communication styles, the fourth intercultural areas, and the fifth focused on intercultural abilities (i.e., knowledge, attitude, skills, and awareness of cultural differences). The total number of questions was 109 .

\section{NIC-test}

The first section of the NIC questionnaire contained a demographic chapter, in which all the information relevant to the participant was obtained, the second section focused on personal characteristics, and the third section focused on communication styles. The total number of questions was 68 . 


\section{Semistructured interviews with NIC}

In addition to the questionnaires, semistructured interviews were conducted with three Nepalese rescuers and three Nepalese physicians, who were randomly selected 10 weeks after the end of the course. These semistructured interviews were conducted in Nepalese, through telephone. The team member responsible for this task (M.P.) was a previous NIC who after completing the course became a member of the research group. The oral responses were transcribed into English and entered partly as assessment of intercultural competence of the WIs by NICs and partly as qualitative data.

\section{Statistical analysis}

The results of the questionnaires were described with mean \pm SD or range where appropriate, otherwise percentages and absolute numbers were indicated. Association between nominal variables in general was tested by using chi-square test. In the case of nominal variables with two categories, Fisher's exact test was preferred. We used the z-test to compare column proportions. For the z-test, each subscript letter denotes a subset of categories whose column proportions do not differ significantly from each other at the 0.05 level. To explore the differences between two groups for continuous variables, either the Kruskal-Wallis test or the independent samples test for equality of means ( $t$-test) was used, in accordance with the distribution of the data. $p<0.05$ was considered significant in all analyses.

\section{Personal characteristics of WIs and NICs}

WIs and NICs were required to rate their own personal characteristics using a Likert scale from 0 (lowest score) to 5 (highest score) in relation to a list of 15 adjectives (Table 1), corresponding to their self-perception in their own culture. To quantify the difference in response between the two groups with regard to their perceived personal characteristics, an extra variable was created by summing the rates of all 15 questions for each respondent. Afterward, an independent $t$-test for equality of means was applied.

\section{Communication styles}

To explore different communication styles and behaviors of WIs and NICs, Fisher's exact test and associations between being WI or NIC were used.

\section{WIs' intercultural abilities}

To explore intercultural abilities of WIs, two groups of questions on knowledge and attitude were evaluated. The respondents had to rate themselves on each item from the corresponding questionnaires using a Likert scale from 0 (lowest

Table 1. List of Adjectives fOR Self-Description

\begin{tabular}{ll}
\hline Intolerant & Tolerates ambiguity \\
Flexible & Open minded \\
Patient & Motivated \\
Lacks sense of humor & Self-reliant \\
Tolerates differences & Empathic \\
Suspends judgment & Clear sense of self \\
Adaptable & Perceptive \\
Curious & \\
\hline
\end{tabular}

score) to 5 (highest score). The same questions were asked in the pre- and postcourse WI questionnaires. To measure differences in mean of the intercultural abilities, two extra variables were created by summing for each respondent the scores for all questions, respectively, for the sections knowledge and attitude separately. Subsequently, the paired samples $t$-tests for equality of means were applied to control whether the self-evaluation of WIs changed before and after the course. To quantify the difference in mean of the self-assessment of cultural abilities of WIs and how they were perceived in Nepal by the NICs in the semistructured interviews, an independent $t$-test for equality of means was applied.

\section{Qualitative data of the semistructured interviews}

We divided the responses into three areas: skills, attitude, and knowledge. We then made a list of substantive words in the answers, with respective frequencies. We selected the terms with frequencies $\geq 3$ and built the word clouds to graphically illustrate keywords arising from the semistructured interviews. In each word cloud, the shading of the term is assigned randomly and the size is related to the term's frequency. The higher the frequency of the term, the larger its size in the word cloud. The preprocessing procedures were performed using the package "tm" in R (Feinerer, 2017). The word clouds were obtained using the Wordle applet (Feinberg, 2014).

\section{Results}

\section{Demographics}

The sample consisted of 18 NICs and 9 WIs. All 18 NICs consented to the study and answered the questionnaire. Out of the nine WIs, one refused consent and eight agreed to take part in the study.

Of the eight WIs, six were Italians, one German, and one Slovenian. Six WIs declared German as their native language, one declared Italian, and another Slovenian. Additional language knowledge can be found in Table 2, and educational profiles in Table 3 . The group was made up of seven men and one woman. Five WIs declared affiliation to the Roman Catholic religion and three did not answer the question. The average age of WIs was $45.9 \pm 9.7$ years (minimum 33, maximum 57).

Table 2. Language Knowledge of Western InSTRUCTORS AND NEPALESE INSTRUCTOR CANDIDATES Besides Mother Tongue

\begin{tabular}{llc}
\hline Participants & \multicolumn{1}{c}{ Language } & Frequency \\
\hline Western instructors (WIs) & English & 6 \\
& Italian & 3 \\
& Serbo-Croatian & 1 \\
& French & 1 \\
& Japanese & 1 \\
Nepalese instructor & English & 18 \\
candidates (NICs) & Hindi & 9 \\
& Nepali & 2 \\
& Sherpa & 2 \\
& Tibetan & 2 \\
& Chinese & 1 \\
& French & 1 \\
& Spanish & 1 \\
& Dutch & 1 \\
& Finnish & 1 \\
\hline
\end{tabular}


Table 3. Educational Profiles of Western Instructors and Nepalese Instructor Candidates

\begin{tabular}{clcc}
\hline & & $\begin{array}{c}\text { Western } \\
\text { instructors }\end{array}$ & $\begin{array}{c}\text { Nepalese instructor } \\
\text { candidates }\end{array}$ \\
\hline $\begin{array}{l}\text { Affiliation to education profile } \\
\text { (only one may apply) }\end{array}$ & No formal education & 0 & 0 \\
& Primary school & 0 & 1 \\
& Secondary school & 3 & 3 \\
& 2 years college & 1 & 2 \\
Affiliation to professional profile & College/University (4 years) & 0 & 9 \\
(multiple choices possible) & Master & 1 & 3 \\
& Doctorate & 3 & 0 \\
& Student & 0 & 0 \\
& Educatian & 3 & 6 \\
& Mountain guide & 4 & 3 \\
& Mountain rescuer & 2 & 4 \\
\end{tabular}

All NICs were of Nepalese nationality. Sixteen declared Nepali as their native language, one Nepali-Sherpa, and one other did not declare any language affiliation. For additional language knowledge, see Table 1. All NICs were men. The group of NICs was characterized by eight people of Hindu religion, seven Buddhists, two Hindu-Buddhists, and one agnostic person. The average age of NICs was $32.8 \pm 7.3$ years (minimum 24, maximum 50).

\section{Self-perception of WIs and NICs}

There is no significant difference in mean of the perception of "self in your own culture" between WIs and NICs $(t=0.687, p=0.499)$.

\section{Communication styles of WIs and NICs}

When meeting people in their own culture (question 3), 6 of 8 WIs and 18 of 18 NICs preferred to get to know each other well before getting down to business, the rest preferred to get down to business as soon as possible (Fisher's exact test $=0.086, p<0.05)$. In a conflict situation in their own culture (question 5), half of WIs preferred exchanges that are dispassionate, and the other half declared they would want people to reveal their true feelings and emotions, whereas nearly all (17 of 18, 1 missing response) of the NICs declared a preference for people to reveal their true feelings and emotions. (Fisher's exact test $=0.006, p<0.05$ ). However, when disagreeing in their own culture (question 15), nearly all of the WIs (7 of 8) and half of the NICs (9 of 18) preferred to be told directly and openly about the problem no matter the consequence. Alternatively, one WI and half of NICs indicated that they would prefer not to speak openly so as not to offend anyone (Fisher's exact test $=0.099, p<0.05$ ). When negating someone's comment or request in their culture (question 21), WIs preferred to say so directly and unambiguously (six of eight). NICs preferred to convey this without saying so directly (12 of 18) (Fisher's exact test $=0.09$, $p<0.05)$. If foreigners speak the language of the respondent (question 33), five of eight WIs would treat them differently, and three WIs generally treated them the same as themselves. NICs would generally treat them the same as themselves (13 of 17) (Fisher's exact test $=0.087, p<0.05$ ). When meeting people from different cultures, 2 of 7 WIs and 12 of 17 NICs admitted that they adjust their type of eye contact (question 40) according to whether an individual is from a different cultural background or not (Fisher's exact test $=0.085$, $p<0.05)$. All other results did not reach statistical significance.

\section{Change of the intercultural abilities of WIs}

According to the results for the section "knowledge" of WIs, we did not find a significant difference in mean before and after the course $(t=0.293, p=0.778)$. However, there was a trend for WIs to change their prior attitude toward the host culture after the course $(t=-2.278, p=0.057)$. In addition, we did not find a significant difference between the WIs' selfperception of their intercultural abilities compared with the assessment of the WIs by the NICs $(t=0.307, p=0.769)$.

\section{Semistructured interviews}

Semistructured interviews with six NICs (three rescuers and three physicians) highlighted the importance of language, time, and dedication of those instructors who commit to delivering educational courses abroad. The descriptive words used by NIC respondents are arranged according to their frequency distribution, and depicted as three word clouds representing the aspects of knowledge, skills, and attitude identified (Figs. 1-3). The NICs often described that in their opinion, the WIs did not have enough time to get to know their host culture. One of the NICs said, “...The instructors were too busy to learn about our behaviors, social skills, and cultural aspects. They hardly had any time to

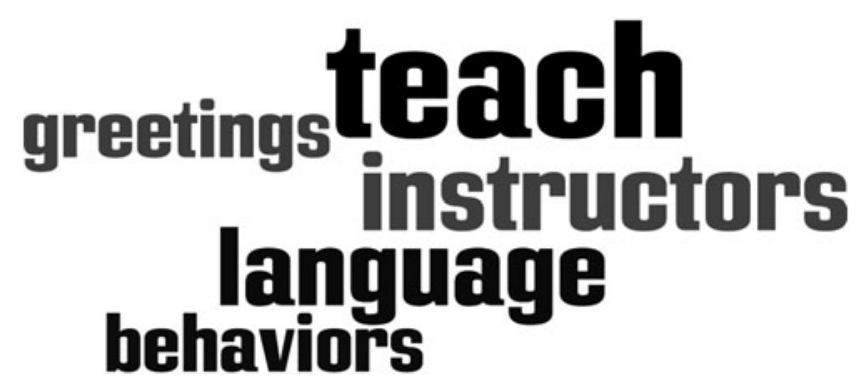

FIG. 1. Word cloud "Knowledge" with frequency $\geq 3$. 


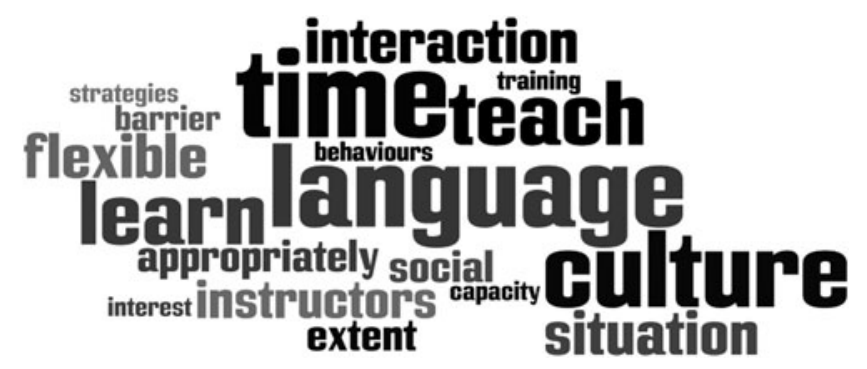

FIG. 2. Word cloud "Skills" with frequency $\geq 3$.

interact and understand our culture, language, and behavior." Language was an issue, but "Despite English being second language for all of us (NICs), they (WIs) dealt pretty well." Moreover, prior exposure with the host culture clearly was an advantage: "... There was difference between the instructors who had already been in Nepal versus those who were here for the first time. Those who had previous visits in Nepal obviously knew those essential norms and taboos to some extent...."

\section{Discussion}

This study on intercultural competence of WIs teaching NICs was conducted during the first internationally led instructor course for mountain rescuers and mountain rescue doctors in Nepal in 2015. It showed that there were differences in communication styles between WIs and NICs, which could influence teaching and learning experiences.

Personal characteristics of these two groups were similar in the self-assessment, and the intercultural competence selfassessment of the WIs corresponded well with the assessment of the WIs completed by the NICs. The main difference was found in communication styles between WIs and NICs in "conflict" situations: NICs mainly want to build or improve a relationship with an individual before entering into a conflict or difficult situation, whereas, independent of relationship status, WIs prefer to deliver criticism directly and "put the facts on the table." If NICs must say something critical, they tend to avoid direct confrontation and prefer to "skate around" the topic. These differences could have a substantial impact on teaching and learning: conflict avoidance, for example, could potentially lead to suboptimal utilization of constructive feedback. Modern educational methods, in-

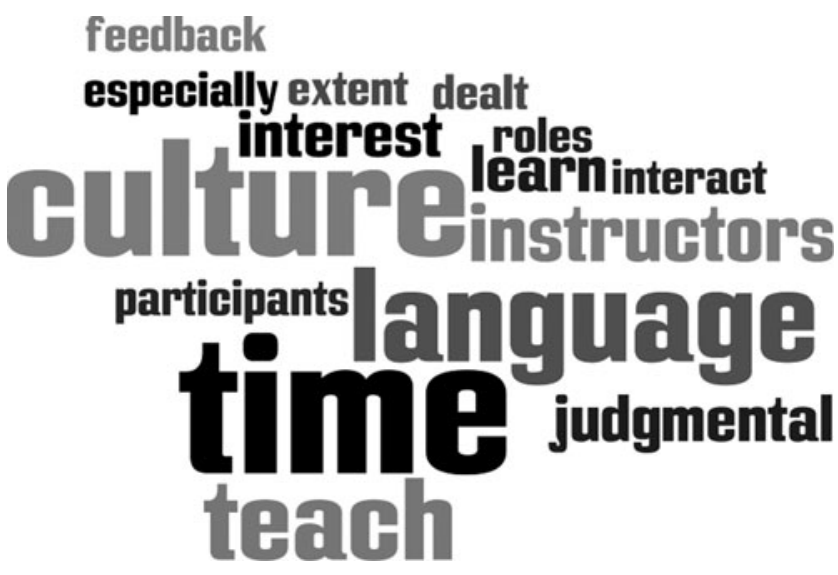

FIG. 3. Word cloud "Attitude" with frequency $\geq 3$. cluding problem-based learning and constructive feedback sessions, still are almost nonexistent even in high-level academic institutions in Nepal. Providing constructive feedback to Nepalese students in a way that facilitates effective learning, therefore, is a very sensitive and challenging topic for instructors with different cultural backgrounds.

Besides assessing communication styles of WIs and NICs, we were interested to see whether the intercultural competence of the WI group changed during their stay in Nepal. In the pre-post self-assessment, we were surprised to find that even though WIs did not improve their knowledge of the host culture, they seemed to change their attitude toward it. In the semistructured interviews with the six NICs, they stated that the WIs did not have enough time to be exposed to the $\mathrm{Ne}$ palese culture while fulfilling their teaching commitment. Therefore, the observed trend to changing attitude is even more surprising. NICs stated that they had seen differences in the behavior of the WIs and that WIs with prior exposure to Nepalese culture were more aware of local cultural norms.

The results of this study highlight potential differences in teaching and learning between cultures. Responses from Nepalese participants indicate that knowledge of cultural norms and preferred communication styles is important to avoid misunderstandings and ineffective educational experiences. Additional effort should be directed toward the way the message is conveyed with less emphasis on the content of the message: the method of teaching, the level of interaction, and the sensitivity of the faculty are at least as important as the course content.

Faculty should either have prior cultural awareness or program-led opportunities to better understand educative norms in host countries. Watling et al. (2013) found that credibility and constructiveness were described as essential characteristics for teachers in intercultural settings to make feedback effective. Participants need to perceive their teachers as fair, accurate, and legitimate especially when they receive a critical feedback. In addition, some of the most important competencies for intercultural educators are reported to be simply being knowledgeable, respectful, and empathic. In addition to the preparation of faculty members before their intercultural exchange, teachers should allocate time for direct interaction with the host culture, tradition, and environment during their stay. This would not only reinforce their intercultural competence, but could also ensure that their students are more at ease and able to learn effectively.

\section{Limitations}

Despite the unique educational scenario and respondents, the study design employed within this project has limitations. Because the sample size is small, including just 8 WIs and 18 NICs, the study lacks statistical power. The study design could not rely on prior similar research projects, as literature is scarce regarding Western experiences when delivering educational programs abroad. We had to adapt a questionnaire that best fit our needs. Throughout the data collection period, we faced considerable language difficulties for our study: for only one WI and for no NIC, English was the native language. We tried to overcome this challenge by assisting all the study participants in their native language when they had to complete the questionnaires. Moreover, all NICs' semistructured interviews were conducted in Nepalese and later translated into English. 


\section{Conclusion}

In this cohort study on intercultural competence of WIs teaching Nepalese rescuers and rescue doctors during the first instructor course in Nepal, we we were able to show differences in communication style between WIs and NICs that might be relevant in medical education. For future work in intercultural settings, teachers and students should be sensitized to differences in communication style. They should be prepared to create a constructive learning environment, which respects cultural diversity and, therefore, optimizes learning. In addition, faculty members should have time and opportunities to become acquainted with the host culture. Intercultural competence can be fostered by preparation and a respectful attitude toward the host culture. If the participants are well prepared, such encounters can become a unique and unforgettable experience.

\section{Acknowledgments}

Without the active participation of the Western faculty and the Nepalese course participants, this research would not have been possible. We thank them for their participation. We also thank the International Commission for Alpine Rescue (ICAR) and the EURAC Institute of Mountain Emergency Medicine for their support of the study.

\section{Author Disclosure Statement}

The authors of the study do not have any financial conflict of interest to report.

\section{References}

Al Ansari A, Al Khalifa K, Al Azzawi M, Al Amer R, Al Sharqi D, Al-Mansoor A, and Munshi FM. (2015). Cross-cultural challenges for assessing medical professionalism among clerkship physicians in a middle eastern country (Bahrain): Feasibility and psychometric properties of multisource feedback. Adv Med Educ Pract 6:509.

Alizadeh S, Chavan M. (2016). Cultural competence dimensions and outcomes: A systematic review of the literature. Health Soc Care Community 24:e117-e130.

Deardorff DK. (2006). Identification and assessment of intercultural competence as a student outcome of internationalization. J Stud Int Educ 10:241-266.

Deardorff DK. (2009). The SAGE Handbook of Intercultural Competence. Sage, Los Angeles, CA.

Drain PK, Primack A, Hunt DD, Fawzi WW, Holmes KK, and Gardner P. (2007). Global health in medical education: A call for more training and opportunities. Acad Med 82:226-230.

Ellerton J, Tomazin I, Brugger H, and Paal P. (2009). Immobilization and splinting in mountain rescue: Official recommendations of the International Commission for Mountain Emergency Medicine, ICAR MEDCOM, intended for mountain rescue first responders, physicians, and rescue organizations. High Alt Med Biol 10:337-342.

Emert HA, and Pearson DL. (2007). Expanding the vision of international education: Collaboration, assessment, and intercultural development. N Dir Community Colleges 2007:67-75.

Fantini A, and Tirmizi A. (2006). Exploring and Assessing Intercultural Competence. World Learning Publications. Available at: http://digitalcollections.sit.edu/worldlearning_publications/1 (accessed August 15, 2018).

Fantini AE. (2009). Assessing intercultural competence. In: The SAGE Handbook of Intercultural Competence. DK Deardorff, ed. Sage, Los Angeles, CA. pp. 456-476.
Feinberg, J. (2014). Wordle applet. Retrieved from www .wordle.net

Feinerer I. H. K. (2017). tm: Text Mining Package. R package version 0.7-3. Retrieved from https://CRAN.R-project.org/ package $=$ tm

Hamilton J. (2009). Intercultural competence in medical education-essential to acquire, difficult to assess. Med Teach 31: 862-865.

Holmes P, and O'Neill G. (2012). Developing and evaluating intercultural competence: Ethnographies of intercultural encounters. Int J Intercultural Relat 36:707-718.

Jha V, Mclean M, Gibbs TJ, and Sandars J. (2015). Medical professionalism across cultures: A challenge for medicine and medical education. Med Teach 37:74-80.

Kratzke C, and Bertolo M. (2013). Enhancing students' cultural competence using cross-cultural experiential learning. J Cult Divers 20:107-111.

Maeder MB, and Pun M. (2018). Medical aspects of the Gorkha earthquake 2015: Disaster preparedness and response. In: Living Under the Threat of Earthquakes. JH Kruhl, R Adhikari, and UE Dorka, eds. Springer, Cham, Switzerland. pp. 155-173.

Maeder MM, Basnyat B, and Harris NS. (2014). From Matterhorn to Mt Everest: Empowering rescuers and improving medical care in Nepal. Wilderness Environ Med 25:177-181.

Sinicrope C, Norris J, and Watanabe Y. (2007). Understanding and assessing intercultural competence: A summary of theory, research, and practice (technical report for the foreign language program evaluation project). University of Hawai' I Second Langauge Studies Paper 26.

Strapazzon G, Ponchia A, Ellerton J, and Brugger, H. (2011). Risk assessment and emergency management of coronary heart disease at altitude. High Alt Med Biol 12:97-98.

Thapa GB, Neupane M, Strapazzon G, Basnyat B, Elsenshon F, Brodmann Maeder M, and Brugger H. (2014). Nepalese mountain rescue development project. High Alt Med Biol 15:91-92.

Tomazin I, Ellerton J, Reisten O, Soteras I, Avbelj M, and International Commission for Mountain Emergency, $\mathrm{M}$. (2011). Medical standards for mountain rescue operations using helicopters: Official consensus recommendations of the International Commission for Mountain Emergency Medicine (ICAR MEDCOM). High Alt Med Biol 12:335-341.

Volet S. (2004). Challenges of internationalisation: Enhancing intercultural competence and skills for critical reflection on the situated and non-neutral nature of knowledge. Lang Acad Skills Higher Educ 6:1-10.

Watling C, Driessen E, Vleuten CP, Vanstone M, and Lingard L. (2013). Beyond individualism: Professional culture and its influence on feedback. Med Educ 47:585-594.

Zafren K, Brants A, Tabner K, Nyberg A, Pun M, Basnyat B, and Maeder MB. (2018). Wilderness mass casualty incident (MCI): Rescue chain after avalanche at Everest Base Camp (EBC) in 2015. Wilderness Environ Med 29:401-410.

Address correspondence to: Monika Brodmann Maeder, MD, MME Institute of Mountain Emergency Medicine EURAC Research Viale Druso 1 Bolzano 39100 Italy

E-mail: monika.brodmann@eurac.edu

Received August 23, 2018; accepted in final form October 1, 2018. 